\title{
Long-Term Follow Up of Secundum Atrial Septal Defect Closure with the Amplatzer Septal Occluder
}

\author{
Marc D. Knepp, MD, Albert P. Rocchini, MD, Thomas R. Lloyd, MD, and Ranjit M. Aiyagari, MD \\ University of Michigan Congenital Heart Center-Department of Pediatrics, Ann Arbor, Mich., USA
}

\begin{abstract}
A B S T R A C T
Background. During the past 15 years, closure of a secundum atrial septal defect (ASD) has moved from a surgical to a percutaneous transcatheter approach. Few long-term studies of the efficacy and safety of closure of an ASD by an Amplatzer septal occluder (ASO) exist.

Methods. To examine the long-term results of secundum ASD closure using the ASO, data on 94 patients who underwent secundum ASD closure with the ASO between 1998 and 2002 were available and reviewed. Data regarding residual shunt, chest pain, palpitations, arrhythmias, headaches, transient ischemic attacks, cerebrovascular accidents, and mortality were collected.

Results. Seven (7.4\%) subjects had residual shunts immediately following ASO placement. During follow-up, 4 residual shunts closed for a complete closure rate of $97 \%$. Eighteen (19\%) patients reported chest pain during the follow-up period. Twenty-three patients (24\%) reported palpitations during the follow up period, 7 were documented arrhythmias, including supraventricular tachycardia, atrial fibrillation, and premature ventricular beats. Migraine headaches were new-onset in 4 patients. Migraine cessation occurred in 2 patients after secundum ASD closure. One child died from a cerebral vascular event 18 months following device placement. Only 1 patient developed mild aortic insufficiency.

Conclusion. These data indicate that for up to 120 months of patient follow-up, the ASO continues to be a safe device. Residual shunts and arrhythmias have low incidence post-ASO placement. Given the mortality in one high-risk patient, further investigation into anti-platelet therapy after device placement is warranted.
\end{abstract}

Key Words. Amplatzer Septal Occluder; Atrial Septal Defect; Palpitations

\section{Introduction}

$\mathrm{D}$ uring the past 15 years, closure of secundum atrial septal defects (ASD) has moved from a surgical approach in the operating room to a percutaneous transcatheter approach in the cardiac catheterization laboratory. ${ }^{1}$ Reports of transcatheter secundum ASD closure studies have shown improvement in cardiac dimensions and function, as well as right ventricular systolic pressure and pulmonary artery hypertension..$^{2-4}$ In contrast to surgical closure, the transcatheter approach avoids cardiopulmonary bypass, results in shorter hospitalization, reduces need for blood products, lessens patient discomfort, produces similar outcomes, and decreases costs. ${ }^{5-8}$ One such device used for transcatheter closure, the Amplatzer septal occluder (ASO), was initially used in humans in
1995. ${ }^{9}$ Short- and intermediate-term studies have shown the device to be safe and effective. ${ }^{10,11}$ Given that few long-term studies of the ASO exist, ${ }^{12}$ and complications after device placement continue to be reported, ${ }^{13-15}$ this report summarizes long-term secundum ASD closure outcomes using the ASO.

\section{Methods}

From our pediatric catheterization laboratory device database, 159 patients who had undergone secundum ASD closure with the ASO between 1998 and 2002 were identified. Ninety-four patients were pediatric ( $\leq 18$ years of age) and 65 were adults ( $\geq 19$ years) at the time of device placement. The study was approved by the institutional
Journal Compilation () 2010 Wiley Periodicals, Inc. 
review board. If the patient did not receive follow-up care at our institution, a HIPAA (Health Insurance Portability and Accountability Act)compliant release of information was sought for outside records to be reviewed. Of the 159 patients, 7 patients chose not to be included in the study, 29 patients were lost to follow up, and 29 patients did not respond to requests. The overall response rate was $59 \%$. The study population then comprised of 94 patients (52 pediatric, 42 adult); each subject's medical record was reviewed from presentation at our institution through March of 2008. The following outcomes were collected: residual shunt, chest pain, palpitations, arrhythmias, headaches, transient ischemic attacks (TIA), cerebrovascular accidents (CVA), and mortality. Mean age at implantation for pediatric patients was $7.5 \pm 4.6$ years, and for adults was $49.9 \pm 15.4$ years.

All patients had a physical examination, ECG, and echocardiogram prior to device placement. The Amplatzer septal occluder (AGA Medical, Golden Valley, MN) was used exclusively in the group studied. Technical delivery of the device to the atrial septum has been previously described. ${ }^{\text {? }}$ All defects were measured using the balloon stretched diameter technique. Intra-procedural heparin and antibiotics were given to all patients. After device placement, patients received 6 months of aspirin therapy. All had repeat physical

Table 1. Prevalence of residual shunts after ASO placement

\begin{tabular}{lllll}
\hline & $<1$ month & $1-12$ months & $1-5$ years & $>5$ years \\
\hline Pediatric & $2(4 \%)$ & $2(4 \%)$ & $1(2 \%)$ & $1(2 \%)$ \\
Adult & $5(12 \%)$ & $4(10 \%)$ & $2(5 \%)$ & $2(5 \%)$ \\
Total & $7(7 \%)$ & $6(6 \%)$ & $3(3 \%)$ & $3(3 \%)$ \\
\hline
\end{tabular}

Time period after device placement is listed with the corresponding number of residual shunts. examinations, ECG, and echocardiograms 6 months after device placement. Further follow-up and surveillance testing were determined by the patient's primary cardiologist.

Data are expressed in mean \pm standard deviation or median with range as appropriate.

\section{Results}

Median follow up was 73 months (range 63-120). There were 7487 patient-months of follow up. Mean secundum ASD size by balloon stretched diameter was $18.0 \pm 5.7 \mathrm{~mm}$. Mean size of ASO used was $19 \pm 6 \mathrm{~mm}$, yielding a mean device to stretched defect ratio of $108 \%$. In the pediatric group, $16(31 \%)$ received devices with diameters greater than $1 \mathrm{~mm} / \mathrm{kg}$ body weight, and one child had a device diameter greater than $2 \mathrm{~mm} / \mathrm{kg}$.

A total of $7(7.4 \%)$ residual shunts were present immediately following ASO placement. However, in the follow-up period 4 residual shunts closed for a complete closure rate of $97 \%$ (Table 1). The remaining residual shunts were not hemodynamically significant on echocardiography.

Eighteen (19\%) patients reported chest pain during the follow-up period (Table 2). No complaints of chest pain were reported in the first month. All patients with chest pain received workup; only 2 patients were identified with cardiac sources of the chest pain. One pediatric patient had supraventricular tachycardia (SVT) diagnosed by ECG. This occurred 25 months post-device placement. One adult patient had hemorrhagic pericarditis 9 months after device placement. No erosion of the device was noted by physical examination, chest $\mathrm{x}$-ray, or echocardiogram. The patient underwent uncomplicated pericardial window and drainage with the device left in place and recovered completely.

Table 2. Incidence of chest pain, palpitations, and arrhythmias after placement of Amplatzer septal occluder. Each column represents the incidence of the symptom occurring during the time period after device placement.

\begin{tabular}{llllll}
\hline Outcome & & $<1$ month & $1-12$ months & $1-5$ years & $>5$ years \\
\hline Chest Pain & Pediatric & $0(0 \%)$ & $2(4 \%)$ & $4(8 \%)$ & $5(10 \%)$ \\
& Adult & $0(0 \%)$ & $3(7 \%)$ & $3(7 \%)$ & $3(7 \%)$ \\
& Total & $0(0 \%)$ & $5(5 \%)$ & $7(7 \%)$ & $8(15 \%)$ \\
Palpitation & Pediatric & $0(0 \%)$ & $1(2 \%)$ & $5(10 \%)$ & $2(4 \%)$ \\
& Adult & $5(12 \%)$ & $7(17 \%)$ & $3(7 \%)$ & $0(0 \%)$ \\
& Total & $5(5 \%)$ & $8(15 \%)$ & $8(15 \%)$ & $2(2 \%)$ \\
Arrhythmia & Pediatric & $0(0 \%)$ & $0(0 \%)$ & $1(\mathrm{SVT})(2 \%)$ & $1\left(1^{\circ} \mathrm{AVB}\right)(2 \%)$ \\
& Adult & 2 (Afib, SVT) (5\%) & $2($ Afib, PVC) (5\%) & $1($ Afib) (2\%) & $0(0 \%)$ \\
& Total & $2(2 \%)$ & $2(2 \%)$ & $2(2 \%)$ & $1(1 \%)$ \\
\hline
\end{tabular}

Afib, atrial fibrillation, PVC, premature ventricular complexes, SVT, supraventricular tachycardia, $1^{\circ} \mathrm{AVB}$, first degree atrioventricular block. 
Prior to device placement, 14 patients (15\%) had documented arrhythmias. This included three pediatric patients with SVT, none of whom had recurrence of SVT in follow up. One adult had SVT, which did not recur after device placement. Ten adult patients, all of whom were over 40 years of age at the time of device placement, had prior atrial fibrillation. Eight of these 10 adults continued to have atrial fibrillation in the follow up period.

Twenty-three patients (24\%) reported palpitations during the follow up period (Table 2). However, only 7 (7\%) patients had documented new-onset arrhythmias (Table 2). In the pediatric subset, one child had SVT, as noted in the section regarding chest pain above. This child had a presenting symptom of chest pain, but did not report palpitations. She did not have evidence of preexcitation. The child had two more episodes of SVT over the next two months; she was started on digoxin with no more episodes in the following 5 -year period. One child was noted to have firstdegree atrioventricular block found incidentally on a surveillance ECG. Three (7\%) adults developed new-onset atrial fibrillation. Another adult had a first-time, single episode of SVT 2 weeks following device placement. Another had frequent premature ventricular complexes on work-up; these had not been documented prior to device placement.

Migraine headaches were new-onset in 4 (4\%) patients during the follow up period. Conversely, 2 (2\%) patients who had suffered from migraine headaches prior to secundum ASD closure with the ASO did not have a migraine headache during the follow up period. Though three adults had TIAs or CVAs prior to ASO placement, no adults had further neurologic events. One child had a fatal CVA 18 months following device placement.

Review of the echocardiographic data showed that 77 patients $(82 \%)$ had mild dilation of the right sided chambers at time of device implantation. Additionally, nine patients $(10 \%)$ had moderate right sided chamber dilation, while eight patients $(8 \%)$ had no chamber enlargement. During the follow-up period, 37 of the 44 pediatric patients $(84 \%)$ with mild right-sided chamber dilation had resolution to normal chamber dimensions. Nineteen of the 33 adult patients (58\%) had resolution of mild right sided dilation. However, three adults with mild dilation had worsening to moderate dilation during the follow up period. All three of these patients were over the age of 60 at time of device implantation. Lack of a change in right-sided dilation after device implantation was less common in the pediatric subset [ 9 out of 52 $(17 \%)$, compared to the adult subset [13 out of 42 $(31 \%)$.

No pediatric patient developed aortic insufficiency after placement of the ASO. One (2\%) adult patient developed mild aortic insufficiency evident only by echocardiography in the follow up period. In addition, no perforations occurred during the device implantation. One child did have an atrial mass on follow-up echocardiogram (see description below), and no other patients had thrombi or masses identified during the follow up period.

\section{Discussion}

Our study showed that use of the ASO resulted in very high incidence $(97 \%)$ of complete closure. Among rare cases of residual shunt, the majority closed spontaneously. Though recent reports have suggested that transthoracic echocardiography was sufficient to determine ASO size, ${ }^{16}$ all devices in our study were chosen based on defect stretched diameter. This method likely contributed to our low rate of residual shunts.

Chest pain was a common complaint throughout the follow-up period. Although the complaint was common, a cardiac etiology was rare. Interestingly, no patient complained of chest pain during the first month after ASO placement. This observation may be related to the counseling that patients received prior to device placement, minimizing potential concerns a patient may have in the immediate post-placement period. This may have led to under-reporting of symptoms. One patient had chest pain due to a tachyarrhythmia, a known cause of chest discomfort.

Similarly, palpitations were reported quite infrequently in pediatric patients. Adults, conversely, complained more frequently of palpitations earlier in the post-placement period. Again, pre-device counseling may have led to underreporting. However, only $25 \%$ of children with complaints of palpitations had a documented arrhythmia.

Several patients had documented arrhythmias prior to device implantation. Interestingly, none of the pediatric patients had recurrence of arrhythmia after device placement. Conversely, nearly $80 \%$ of the adult patients had recurrence of their atrial fibrillation after device placement. This appears to be very similar to what has been described in the adult surgical literature, ${ }^{17,18}$ in 
which data suggest that patients who are older at the time of intervention are more likely to have recurrence of atrial arrhythmias. All of our adult patients who had recurrence of their atrial arrhythmias were older than 50 at the time of implantation, except one patient who was 41 years old. The 3 of 11 adult patients that did not have recurrences were 47,63 , and 79 years of age.

In addition, $10 \%$ of adults developed a new arrhythmia post-ASO placement. All of these patients were older than 50 years at the time of device placement. This observation, however, may be independent of the ASO. The expected course of a dilated atrium is to develop arrhythmias; ${ }^{19}$ one could then postulate that the development of atrial fibrillation in the adult sub-group was independent of the device being placed.

The pediatric patient with SVT after device implantation did have a mildly dilated right atrium that did not improve after device placement. The child had no residual shunting on follow up echocardiograms and the device was well seated. The SVT began about one month following the placement of the device and, therefore, was concluded to be related to the device.

The rates of preoperative atrial arrhythmias in our series are comparable to published surgical series. In the series by Murphy, ${ }^{20} 19(15 \%)$ of their patients had atrial flutter or fibrillation prior to surgical ASD closure. Thirteen of those 19 patients $(68 \%)$ continued to have atrial arrhythmias post surgical closure, comparable to our rate of $57 \%$ after ASO placement. In the same surgical series, 23\% developed an arrhythmia during the follow-up period. This is higher than our $7 \%$ rate, though the follow up in the surgical series was between 27 and 32 years. As would be expected, patients who were older at the time of ASD had more arrhythmic events.

The development of migraine headaches in our study group was small, as was the resolution of migraine headaches following device placement. Many studies have looked at the association between patent foramen ovale (PFO) and migraine headaches. ${ }^{21,22}$ Prospective trials have also examined PFO closure with an implantable device as a treatment option. ${ }^{23}$ In our study, we only evaluated patients with device closure of secundum ASD. Although there is an overlap between adults with PFO and secundum ASD, it is not possible to make a generalization about the relationship between migraines and ASO closure of secundum ASD.
The case of hemorrhagic pericarditis has many confounding factors. Though it could be assumed that this was directly related to the device implantation, no perforation was noted during the case or in any of the follow up echocardiograms. The follow up echocardiograms had shown the device to be well seated. At the time of surgery, no device erosion was identified by the surgeon. The patient had been using injectible illicit substances and was intoxicated at the time of admission. In addition, culture of the contents of the pericardium grew coagulase-negative staphylococcus.

The lone mortality in our follow up occurred in a child who had a fatal cerebrovascular accident 18 months following device placement. At the time of device placement, he was 25 months old and weighed $9 \mathrm{~kg}$. He had a large ASD, trisomy 21, poor growth, and significant respiratory illnesses requiring multiple hospitalizations. The child had been felt to be high risk for surgical closure. Therefore, he underwent percutaneous closure with an ASO under general anesthesia with transesophageal echocardiographic guidance. The balloon stretched diameter was $18.6 \mathrm{~mm}$. A $20-\mathrm{mm}$ ASO was placed, but resulted in immediate complete heart block; the device was removed and the child resumed sinus rhythm. An $18 \mathrm{~mm}$ ASO was then placed with no residual shunt or arrhythmia. Intraoperative transesophageal echocardiogram revealed the left atrial disc abutting the anterior leaflet of the mitral valve. The mitral valve was not distorted, and no mitral stenosis and trace mitral regurgitation were seen. The child was maintained on aspirin for 6 months post procedure and had normal follow up echocardiograms. Eighteen months after device placement, he developed fever, lethargy, and no movement on the right side. He quickly became obtunded and was sent to the local emergency department. A CT scan of the head demonstrated occlusion of the left internal carotid artery and a left frontal lobe infarct. Echocardiogram found normal biventricular function, no residual shunt, and a $1 \mathrm{~cm} \times$ $0.5 \mathrm{~cm}$ mass in the left atrium attached to the atrial septum and extending to the posterior leaflet of the mitral valve. No significant mitral stenosis or regurgitation was seen. The patient rapidly deteriorated despite neurosurgical intervention, and support was withdrawn. Autopsy demonstrated a left atrial thrombus adherent to the inferior edge of the device overlying the mitral valve leaflet (Figure 1). Other than this region, the device appears to be endothelialized. Thrombus was partially organized with abundant neutrophil 


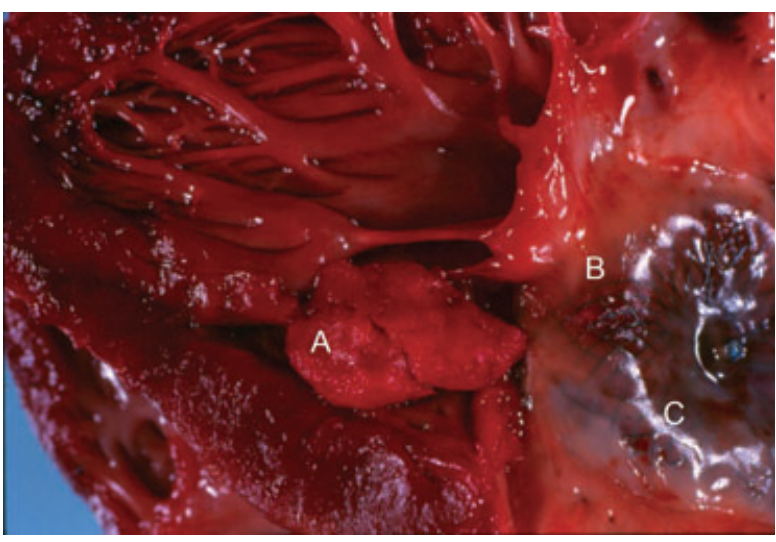

Figure 1. Autopsy photograph of left atrial mass. (A) Thrombus. (B) Thrombus attachment site. (C) Amplatzer septal occluder.

infiltrate, but no infection was identified. It is not known if the child had a hypercoagulable state. Verification of device endothelialization with microscopy was not performed. Although not studied prospectively, the use of aspirin therapy for 6 months has been our protocol for device placement and is based on the amount of time that a majority of ASOs become endothelialized. ${ }^{24,25}$

The limitations of this study include those inherent to retrospective reviews. The overall response rate was only about $60 \%$, which raises the possibility that some adverse events may not have been reported. Due to institutional regulations, further investigation into follow up records is limited. However, given that our institution was the first and only center placing ASOs in our region during the first several years of follow up, it is very likely that any complication would have been brought back to our institution for evaluation.

\section{Conclusion}

These data indicate that for up to 120 months of patient follow-up, the ASO is a safe device for secundum atrial septal defect closure in both pediatric and adult patients. Residual shunts and arrhythmias have low incidence post-ASO placement. The ASO continues to compare favorably to surgical closure. Given the mortality in one high-risk patient, we feel further investigation into anti-platelet therapy after device placement is warranted.

Corresponding Author: Marc D. Knepp, MD, Pediatric Cardiology, University of Michigan, L1242 Women's, SPC 5204, 1500 East Medical Center Drive,
Ann Arbor, MA 48109, USA. Tel: 734-764-5177; Fax: 734-936-9470; E-mail: mknepp@umich.edu

Conflict of interest: None.

Accepted in final form: September 27, 2009.

\section{References}

1 Berger F, Ewert P, Bjornstad PG, et al. Transcatheter closure as standard treatment for most interatrial defects: experience in 200 patients treated with the Amplatzer septal occluder. Cardiol Young. 1999;9:468-473.

2 Schoen SP, Kittner T, Bohl S, et al. Transcatheter closure of atrial septal defects improves right ventricular volume, mass, function, pulmonary pressure, and functional class: a magnetic resonance imaging study. Heart. 2006;92:821-826.

3 Salehian O, Horlick E, Schwerzmann M, et al. Improvements in cardiac form and function after transcatheter closure of secundum atrial septal defects. 7 Am Coll Cardiol. 2005;45:499504.

4 Balint OH, Samman A, Haberer K, et al. Outcomes in patients with pulmonary hypertension undergoing percutaneous atrial septal defect closure. Heart. 2008;94:1189-1193.

5 Cowley CG, Lloyd TR, Bove EL, Gaffney D, Dietrich M, Rocchini AP. Comparison of results of closure of secundum atrial septal defect by surgery versus Amplatzer septal occluder. Am fo Cardiol. 2001;88:589-591.

6 Thomson JDR, Aburawi EH, Watterson KG, Van Doorn C, Gibbs JL. Surgical and transcatheter (Amplatzer) closure of atrial septal defects: a prospective comparison of results and cost. Heart. 2002;87:466-469.

7 Hughes M, Maskell G, Goh T, Wilkinson J. Prospective comparison of costs and short term health outcomes of surgical versus device closure of atrial septal defect in children. Heart. 2002;88:6770.

8 Du ZD, Hijazi ZM, Kleinman CS, Silverman NH, Larntz K. Comparison between transcatheter and surgical closure of secundum atrial septal defect in children and adults. Fo Am Coll Cardiol. 2002;39: 1836-1844.

9 Masura J, Gavora P, Formanek A, Hijazi ZM. Transcatheter closure of secundum atrial septal defects using the new self-centering Amplatzer septal occluder: initial human experience. Cathet Cardiovasc Diagn. 1997;42:388-393.

10 Chan KC, Godman MJ, Walsh K, Wilson N, Redington A, Gibbs JL. Transcatheter closure of atrial septal defect and interatrial communications with a new self expanding nitinol double disc device (Amplatzer septal occluder): multicentre UK experience. Heart. 1999;82:300-306. 
11 Fischer G, Stieh J, Uebing A, Hoffmann U, Morf G, Kramer HH. Experience with transcatheter closure of secundum atrial septal defects using the Amplatzer septal occluder: a single centre study in 236 consecutive patients. Heart. 2003;89:199-204.

12 Masura J, Gavora P, Podnar T. Long-term outcome of transcatheter secundum-type atrial septal defect closure using Amplatzer septal occluders. $7 \mathrm{Am}$ Coll Cardiol. 2005;45:505-507.

13 Chessa M, Carminati M, Butera G, et al. Early and late complications associated with transcatheter occlusion of secundum atrial septal defect. $7 \mathrm{Am}$ Coll Cardiol. 2002;39:1061-1065.

14 Spence M, Qureshi S. Complications of transcatheter closure of atrial septal defects. Heart. 2005; 91:1512-1514.

15 Schoen SP, Boscheri A, Lange SA, et al. Incidence of aortic valve regurgitation and outcome after percutaneous closure of atrial septal defects and patent foramen ovale. Heart. 2008;94:844-847.

16 Li GS, Kong GM, Ji QS, et al. Reliability of transthoracic echocardiography in estimating the size of Amplatzer septal occluder and guiding percutaneous closure of atrial septal defects. Chinese Med $\mathcal{F}$. 2008;121:973-976.

17 Gatzoulis MA, Freeman MA, Siu SC, Webb GD, Harris L. Atrial arrhythmia after surgical closure of atrial septal defects in adults. NEFM. 1999;340:839846.

18 Ghosh S, Chatterjee S, Black E, Firmin RK. Surgical closure of atrial septal defects in adults: effect of age at operation on outcome. Heart. 2002;88: $485-487$.

19 Morton JB, Sanders P, Vohra JK, et al. Effect of chronic right atrial stretch on atrial electrical remodeling in patients with an atrial septal defect. Circulation. 2003;107:1775-1782.

20 Murphy JG, Gersh BJ, McGoon MD, et al. Longterm outcome after surgical repair of isolated atrial septal defect. NE7M. 1990;323:1645-1650.

21 Domitrz I, Mieszkowski J, Kaminska A. Relationship between migraine and patent foramen ovale: a study of 121 patients with migraine. Headache. 2007;47:1311-1318.

22 Anzola GP, Meneghetti G, Zanferrari C, Adami A, Dinia L, Del Sette M. Is migraine associated with right-to-left shunt a separate disease? Results of the SAM study. Cephalalgia. 2008;28:360-366.

23 Dowson A, Mullen MJ, Peatfield R, et al. Migraine intervention with STARFlex technology (MIST) trial. Circulation. 2008;117:1397-1404.

24 Kreutzer J, Ryan CA, Gauvreau K, Van Praagh R, Anderson JM, Jenkins KJ. Healing response to the clamshell device for closure of intracardiac defects in humans. Cathet Cardiovasc Intervent. 2001;54: 101-111.

25 Sharafuddin MJ, Gu X, Titus JL, Urness $M$, Cervera-Ceballos JJ, Amplatz K. Transvenous closure of secundum atrial septal defect: preliminary results with a new self-expanding nitinol prosthesis in a swine model. Circulation. 1997;95:21622168 . 